\title{
Article \\ Explorations on Growth of Blue-Green-Yellow-Red InGaN Quantum Dots by Plasma-Assisted Molecular Beam Epitaxy
}

\author{
Xue Zhang ${ }^{1,2}$, Zhiwei Xing ${ }^{1,2}$, Wenxian Yang ${ }^{2, *}$, Haibing Qiu ${ }^{1,2}$, Ying Gu ${ }^{1,2}$, Yuta Suzuki ${ }^{3}$, Sakuya Kaneko ${ }^{3}$, \\ Yuki Matsuda ${ }^{3}$, Shinji Izumi ${ }^{3}$, Yuichi Nakamura ${ }^{3}$, Yong Cai ${ }^{2}$, Lifeng Bian ${ }^{4}$, Shulong Lu ${ }^{2, *}$ \\ and Atsushi Tackeuchi ${ }^{3}$
}

check for updates

Citation: Zhang, X.; Xing, Z.; Yang, W.; Qiu, H.; Gu, Y.; Suzuki, Y.;

Kaneko, S.; Matsuda, Y.; Izumi, S.; Nakamura, Y.; et al. Explorations on Growth of Blue-Green-Yellow-Red InGaN Quantum Dots by

Plasma-Assisted Molecular Beam

Epitaxy. Nanomaterials 2022, 12, 800 https://doi.org/10.3390/ nano12050800

Academic Editor: Efrat Lifshitz

Received: 8 February 2022

Accepted: 24 February 2022

Published: 26 February 2022

Publisher's Note: MDPI stays neutral with regard to jurisdictional claims in published maps and institutional affiliations.

Copyright: (C) 2022 by the authors. Licensee MDPI, Basel, Switzerland. This article is an open access article distributed under the terms and conditions of the Creative Commons Attribution (CC BY) license (https:// creativecommons.org/licenses/by/ $4.0 /)$.
1 School of Nano-Tech and Nano-Bionics, University of Science and Technology of China, Hefei 230026, China; xzhang2019@sinano.ac.cn (X.Z.); zwxing2017@sinano.ac.cn (Z.X.); hbqiu2018@sinano.ac.cn (H.Q.); ygu2021@sinano.ac.cn (Y.G.)

2 Key Lab of Nanodevices and Applications, Suzhou Institute of Nano-Tech and Nano-Bionics (SINANO), Chinese Academy of Sciences (CAS), Suzhou 215123, China; ycai2008@sinano.ac.cn

3 Department of Applied Physics, Waseda University, Tokyo 169-8555, Japan; yutasuzu@toki.waseda.jp (Y.S.); ar1ak3@toki.waseda.jp (S.K.); see8c3fed78rgl@akane.waseda.jp (Y.M.); i.shinji@akane.waseda.jp (S.I.); the-last-trial@asagi.waseda.jp (Y.N.); atacke@waseda.jp (A.T.)

4 Frontier Institute of Chip and System, Fudan University, Shanghai 200433, China; lfbian2006@sinano.ac.cn

* Correspondence: wxyang2014@sinano.ac.cn (W.Y.); sllu2008@sinano.ac.cn (S.L.)

\begin{abstract}
Self-assembled growth of blue-green-yellow-red InGaN quantum dots (QDs) on GaN templates using plasma-assisted molecular beam epitaxy were investigated. We concluded that growth conditions, including small $\mathrm{N}_{2}$ flow and high growth temperature are beneficial to the formation of InGaN QDs and improve the crystal quality. The lower In/Ga flux ratio and lower growth temperature are favorable for the formation of QDs of long emission wavelength. Moreover, the nitrogen modulation epitaxy method can extend the wavelength of QDs from green to red. As a result, visible light emissions from $460 \mathrm{~nm}$ to $622 \mathrm{~nm}$ have been achieved. Furthermore, a $505 \mathrm{~nm}$ green light-emitting diode (LED) based on InGaN/GaN MQDs was prepared. The LED has a low external quantum efficiency of $0.14 \%$ and shows an efficiency droop with increasing injection current. However, electroluminescence spectra exhibited a strong wavelength stability, with a negligible shift of less than $1.0 \mathrm{~nm}$ as injection current density increased from $8 \mathrm{~A} / \mathrm{cm}^{2}$ to $160 \mathrm{~A} / \mathrm{cm}^{2}$, owing to the screening of polarization-related electric field in QDs.
\end{abstract}

Keywords: InGaN quantum dots; self-assembled growth; green light-emitting diode; plasma-assisted molecular beam epitaxy

\section{Introduction}

In recent years, red-green-blue (RGB) micro light-emitting diode (micro-LED) has been considered a promising display technology with many excellent features such as high luminescent efficiency, quick response, and long lifespan [1,2]. However, there exist two main restricting factors for the development of full-color micro-LED displays. One is the difficulty in the transfer of massive micro-LED chips, and the other one is the fabrication of efficient red micro-LED chips. Currently, commercial red LED chips are made of phosphidebased AlGaInP material. However, red AlGaInP-based LED is not the best option for the RGB micro-LED matrix, because its external quantum efficiency (EQE) would drastically reduce at micro size, and its poor mechanical properties and incompatibility with InGaNbased blue and green LEDs increase the difficulty of the pick-and-place technique [3-5]. In addition, InGaN-based green LED that used multi-quantum wells (MQWs) as active regions has been suffering from the "green gap" and "efficiency droop" problems; they also generally exhibit emission peak wavelength shift and color purity [6-8]. This is mainly due to the poor polarization field strength and crystal quality of InGaN materials with high In 
content, so its efficiency still needs to be improved [9-11]. In this regard, InGaN quantum dots (QDs) have been intensively investigated as an alternative candidate for high efficiency long wavelength light-emitters. Compared with conventional planar structures, InGaN QDs can promote indium (In) incorporation and, thereby, contribute to improving green and red light emitting. Moreover, InGaN QDs have several more excellent advantages including stronger quantum confinement effect, smaller quantum-confined Stark effect (QCSE) and lower dislocation density due to the effective strain relaxation, which are conducive to the enhancement of internal quantum efficiency (IQE) [12-14]. Thus, InGaN QDs could be considered as a potential material for green and red LEDs in full-color micro-LED displays.

The most widely used self-assembled growth method of InGaN QDs is based on Stranki-Krastanov (S-K) mode by metal-organic chemical vapor deposition (MOCVD) and molecular beam epitaxy (MBE), which utilizes lattice mismatch between the epitaxial layer and substrate $[15,16]$. Therefore, the growth window for the achievement of longwavelength self-assembled QDs, that is, the heteroepitaxy of InGaN QDs with high In composition, is very narrow, so it is still a challenge to obtain high quality QDs. Recently, a variety of methods have been adopted to grown red and near-infrared InGaN QDs, including surface pretreatment, introducing the InGaN QW-QD coupled nanostructure, growth interruption method and photoelectrochemical etched quantum dot templates [17-20]. Despite such achievements, there remains many challenges to be conquered for self-assembled InGaN QDs, such as wavelength control and quantum efficiency to meet the requirements of actual device application. In addition, research on all-visible and long-wavelength LEDs has mainly focused on quantum wells [21-23]. Therefore, it is necessary to explore the growth conditions for the formation of InGaN QDs in detail and to optimize the growth method on this basis to obtain long-wavelength InGaN QDs. Herein, self-assembled InGaN QDs with varied emission wavelengths were obtained on GaN-on-sapphire substrates by plasma-assisted molecular beam epitaxy (PA-MBE). We investigated the inherent relationship between morphology and optical properties of InGaN QDs and growth parameters by atomic force microscopy (AFM), photoluminescence (PL) and time-resolved PL (TRPL) measurements, and then derived the phase diagram of InGaN QDs formation. Moreover, the nitrogen modulation epitaxy method was adopted and extended the wavelength of QDs from green to red. Finally, a green InGaN QDs LED that was prepared and exhibited a strong wavelength stability with injection current.

\section{Experiment Details}

\subsection{Self-Assembled Growth of In GaN QDs}

All InGaN QDs samples were grown on c-plane (0001) 3.5- $\mu$ m-thick GaN-on-sapphire substrates by Veeco Gen 20A PA-MBE system equipped with standard Ga and In effusion cells. Active nitrogen $\left(\mathrm{N}_{2}\right)$ was supplied by a radio-frequency plasma cell. The substates were first degreased using standard solvents, and then thermally outgassed in two steps at $200{ }^{\circ} \mathrm{C}$ for $60 \mathrm{~min}$ and followed by $650{ }^{\circ} \mathrm{C}$ for $30 \mathrm{~min}$ to eliminate surface contamination. Prior to InGaN QDs growth, two GaN buffer layers were deposited consisting of a 100-nmthick high-temperature $\mathrm{GaN}$ layer grown at $740{ }^{\circ} \mathrm{C}$ and a 20 -nm-thick low-temperature $\mathrm{GaN}$ layer grown at $650{ }^{\circ} \mathrm{C}$. Afterwards, a series of InGaN QDs samples were grown under different growth conditions. Table 1 list the detailed growth parameters of InGaN QDs including growth temperature $(\mathrm{Tg})$, growth time $(\mathrm{tg})$, source beam equivalent pressure $(B E P)$, radio-frequency power and $\mathrm{N}_{2}$ flux for active nitrogen $\left(\mathrm{N}_{2}\right)$. The group-III element fluxes in BEP were regulated by controlling the temperatures of In and Ga effusion sources. 
Table 1. The growth parameters of all samples.

\begin{tabular}{ccccccc}
\hline Sample & $\mathbf{T g} /\left({ }^{\circ} \mathbf{C}\right)$ & $\mathbf{N}_{\mathbf{2}}$ Flux/Power $(\mathbf{S c c m} / \mathbf{W})$ & $\mathbf{T g} /(\mathbf{m i n})$ & In BEP/(torr) & Ga BEP/(torr) & $\lambda @ \mathbf{R T} /(\mathbf{n m})$ \\
\hline S1 & 610 & $1.5 / 430$ & 7 & $1.2 \times 10^{-8}$ & $4 \times 10^{-9}$ & 463 \\
S2 & 610 & $1.5 / 430$ & 7 & $1 \times 10^{-8}$ & $6 \times 10^{-9}$ & 496 \\
S3 & 610 & $1.5 / 430$ & 7 & $9 \times 10^{-9}$ & $7 \times 10^{-9}$ & 507 \\
S4 & 610 & $1.0 / 400$ & 7 & $1.2 \times 10^{-8}$ & $4 \times 10^{-9}$ & 460 \\
S5 & 610 & $0.6 / 350$ & 7 & $1.2 \times 10^{-8}$ & $4 \times 10^{-9}$ & 462 \\
S6 & 605 & $1.5 / 430$ & 7 & $9 \times 10^{-9}$ & $3 \times 10^{-9}$ & 523 \\
S7 & 595 & $1.5 / 430$ & 7 & $7.5 \times 10^{-9}$ & $2.5 \times 10^{-9}$ & 581 \\
S8 & 605 & $1.5 / 430$ & $4+(30 \mathrm{~s} / 30 \mathrm{~s}) * 3$ & $9 \times 10^{-9}$ & $3 \times 10^{-9}$ & 598 \\
S9 & 605 & $1.5 / 430$ & $4+(15 \mathrm{~s} / 15 \mathrm{~s}) * 6$ & $9 \times 10^{-9}$ & $3 \times 10^{-9}$ & 622 \\
\hline
\end{tabular}

\subsection{Measurements of Surface Morphology and Optical Properties}

The surface morphology of InGaN QDs was characterized by Bruker Dimension ICON AFM. PL measurements were performed at $10 \mathrm{~K}$ or room temperature by tunable Ti-sapphire lasers with an excitation wavelength of $405 \mathrm{~nm}$. TRPL spectra were measured using a $405 \mathrm{~nm}$ Ti-sapphire laser with a pulse width of $100 \mathrm{fs}$ and a repetition frequency of $80 \mathrm{MHz}$, and detected with a Hamamatsu C4334-04 synchro scan streak camera with a time resolution of $15 \mathrm{ps}$.

\section{Results and Discussion}

\subsection{Formation Mechanism of Self-Assembled InGaN QDs by PA-MBE}

The formation of self-assembled InGaN QDs was based on S-K mode, manifesting by a spontaneous morphological transition from a two-dimensional (2D) surface to threedimensional (3D) islands during growth after a certain thickness of InGaN wetting layer on GaN buffer. Reflection high-energy electron diffraction (RHEED) was used to real-time monitor self-forming process of InGaN QDs, as shows in Figure 1. The 2D-3D transition was confirmed by RHEED pattern changing from the streaky- to spotty-like. The result indicates that the formation of InGaN QDs can be achieved in conventional S-K growth method by PA-MBE.

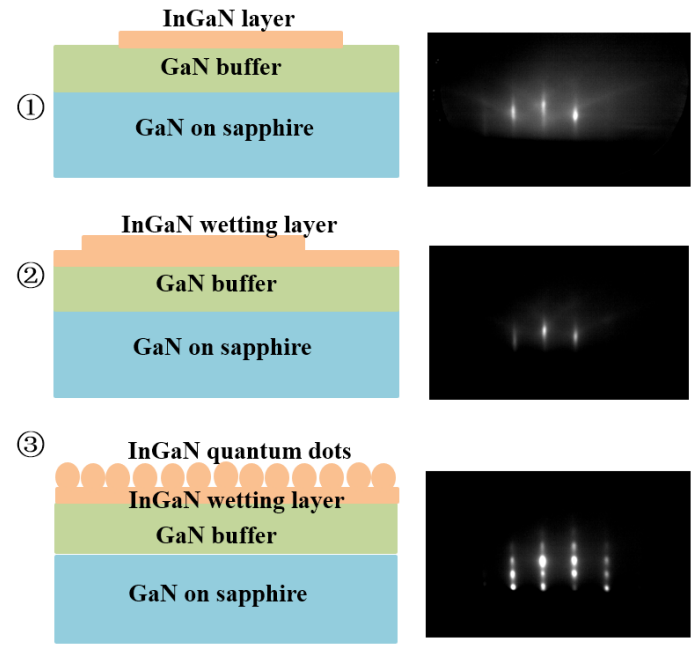

Figure 1. Typical RHEED images of self-assembled InGaN QDs at different growth states.

For InGaN/GaN system, the energy of a 2D surface film and coherently strained 3D islands can respectively be described as [24],

$$
E_{2 D}(h)=E_{2 D}^{e}+E_{2 D}^{S}=M \varepsilon_{I n G a N}^{2} h+\gamma
$$


and

$$
E_{3 D}(h)=E_{3 D}^{e}+E_{3 D}^{S}=(1-\alpha) M \varepsilon_{\text {InGaN }}^{2} h+\gamma+\Delta \gamma
$$

where $M$ is the film's biaxial modulus, $\varepsilon$ is the strain, $h$ is the thickness of $2 \mathrm{D}$ InGaN layer, $E^{e}$ and $E^{s}$ are the elastic energy for InGaN layer and the surface energy for the (0001) surface, respectively. $\alpha$ represents the fraction of $3 \mathrm{D}$ islands covering the surface. $\Delta \gamma$ is the surface energy loss to form the $3 \mathrm{D}$ islands. The layer will undergo a 2D-3D transition and then form InGaN QDs when $E_{3 D}(h)<E_{2 D}(h)$ according to the lowest energy principle. In addition, islanding supposes a sufficient diffusion length that depends mainly on substrate temperature and growth rate. Therefore, it is essential to regulate the film's free energy and facilitate the 2D-3D transition of InGaN by tuning growth parameters such as $\mathrm{N}_{2}$ flow and $\mathrm{Tg}$.

\subsection{Parameter Optimization of In $G a N$ QDs}

The surface morphology of InGaN QDs samples was characterized using an AFM, as shown in Figure 2. The AFM images show uniformly distributed QDs with a high surface density of over $1.0 \times 10^{10} \mathrm{~cm}^{-2}$ for all samples, which indicates that InGaN QDs are obtained by self-assembly method. In order to explore growth of InGaN QDs, the effects of growth conditions on micromorphology and optical properties of InGaN QDs are investigated as follows.

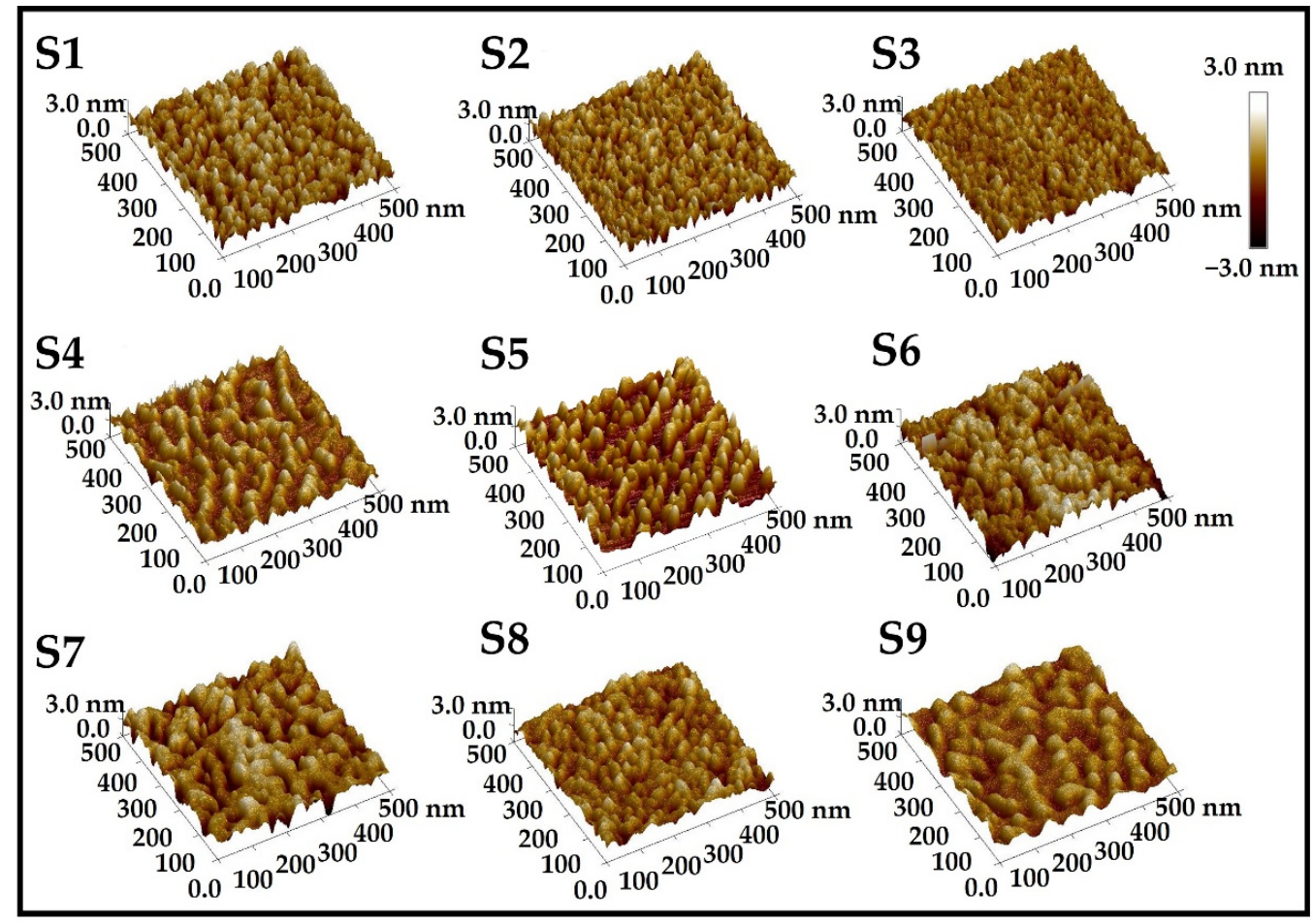

Figure 2. AFM 3D images of InGaN QDs grown under different conditions.

As presented in Table 1, samples S1, S2 and S3 were grown with different In/Ga flux ratio under the premise of constant total group-III metal source, and other growth conditions remain unchanged. Room temperature PL was performed to measure optical properties of InGaN QDs grown under different growth conditions. Figure 3a shows room-temperature PL spectra of S1-S3 samples, and the interference fringes are induced by the Fabry-Perot effect [25]. It is clear that sample S1 has the best optical performance with the strongest PL intensity and the narrowest peak width, which indicates that relatively higher In/Ga ratio can improve the crystal quality of InGaN QDs. In addition, the peak wavelengths of three samples are $463 \mathrm{~nm}, 496 \mathrm{~nm}$ and $507 \mathrm{~nm}$, respectively. That is, the PL peak wavelength of InGaN QDs appears a red-shift with decreasing the In/Ga ratio, 
which is opposite to that of InGaN films. The abnormal behavior of wavelength variation can be explained as the incorporation rate of Ga into InGaN crystal. In general, InGaN material should be grown at a temperature of lower than $650{ }^{\circ} \mathrm{C}$ because of high equilibrium vapor pressure of indium atoms [26]. Such a temperature is enough for In atoms during deposition, but it makes Ga atoms difficult to fully diffuse on the growth front and even incorporate into InGaN crystal under N-rich conditions. With the increase of In/Ga ratio at a given temperature, more In atoms can not only prolong diffusion length and then promote QDs formation, but also enhance the actual incorporation rate of Ga, implying shorter wavelength emission. Therefore, In/Ga flux ratio should be properly reduced to achieve longer wavelength emission, but a reasonable value is needed to ensure the quality of InGaN QDs.

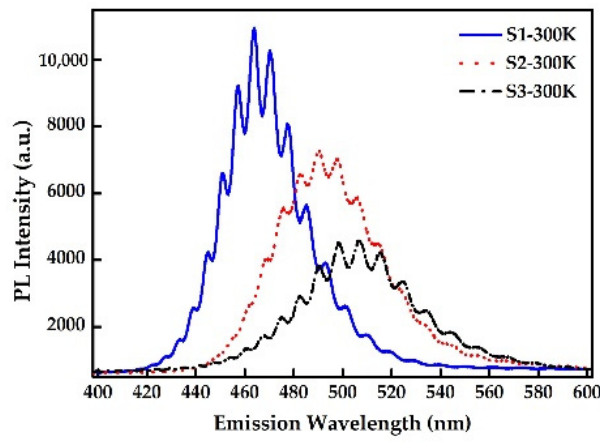

(a)

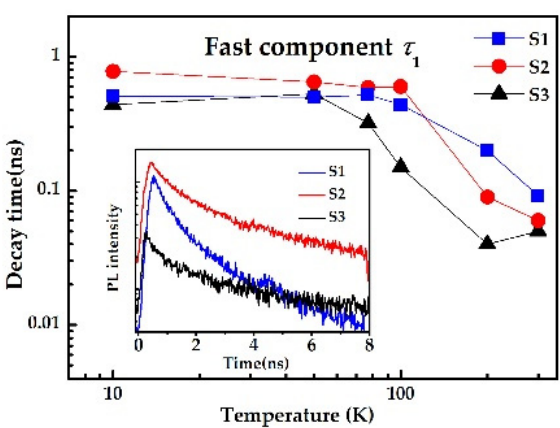

(b)

Figure 3. (a) The PL spectra of S1, S2 and S3 grown with varied In/Ga flux ratio at room temperature; (b) the evolution of fast carrier lifetimes for sample S1, S2 and S3 with temperature. The inset displays $P L$ decay curves at $10 \mathrm{~K}$ under excited power of $3 \mathrm{~mW}$.

Figure $3 \mathrm{~b}$ illustrates TRPL spectra of three samples at $10 \mathrm{~K}$ with an excitation power of $3 \mathrm{~mW}$ and the variations of PL decay times with temperature. All decay curves are well fitted with two-component exponential function [27],

$$
I(t)=A_{1} \exp \left(-t / \tau_{1}\right)+A_{2} \exp \left(-t / \tau_{2}\right)
$$

where $I(t)$ is the PL intensity as a function of time, $A_{1}$ and $A_{2}$ are weighting coefficients, and $\tau_{1}$ and $\tau_{2}$ represent the carrier lifetimes in the fast and slow component, respectively. It is inferred that there exist two-type QD-related localization states because of size and composition fluctuations in self-assembled InGaN QDs. $\tau_{1}$ and $\tau_{2}$ correspond to the slow component in shallow localization and the fast component in deep localization, respectively. By data fitting, it was found that the value of $A_{2}$ was greater or even far greater than $A_{1}$ for all InGaN QDs samples in this work, which implied that the fast component dominated the decay process of photoinduced carriers. In addition, carriers' lifetime as the key performance parameter of semiconductor materials was measured by TRPL, and the spectra were depicted in Figure $3 b$. It was found that carrier lifetimes for both $\tau_{1}$ and $\tau_{2}$ of three InGaN QDs samples decreased with increasing temperature, which was mainly attributed to the thermal activation of more nonradiative recombination centers. By comparison, it was found that sample S1 has the lowest sensitivity to temperature, which means the lowest nonradiative recombination. Therefore, it was further evidence for improving the crystal quality of InGaN QDs by increasing In/Ga flux ratio.

In order to explore the effect of V/III ratio on the formation of InGaN QDs, S1, S4 and $\mathrm{S} 5$ were grown under different $\mathrm{N}_{2}$ flows, as listed in the Table 1 . According to AFM 3D images shown in Figure 2, we can observe that the surface morphologies of samples S4 and S5 are obviously more independent than that of sample S1, which means that high $\mathrm{N}_{2}$ flow is unfavorable to the formation of 3D islands. From Equations (1) and (2), it is clear that low 
$\Delta \gamma$ is conducive to a $2 \mathrm{D}-3 \mathrm{D}$ transition. Considering that $\Delta \gamma$ is in inverse proportional to vacuum pressure, low $\mathrm{N}_{2}$ flux should be applied to improve the morphology of InGaN QDs.

Room-temperature PL spectra of the three QDs samples are represented in Figure 4a. As expected, the PL intensity of InGaN QDs is markedly enhanced by decreasing $\mathrm{N}_{2}$ flux, while the emission wavelength shows no change. In addition, from the TRPL spectra and the variation of fitted decay times with temperature depicted in Figure $4 b$, it is found that lower $\mathrm{N}_{2}$ flux can prolong the decay time of InGaN QDs, and simultaneously reduce its sensitivity to temperature. This is mainly due to the more independent morphology of the self-assembled quantum dots formed at lower $\mathrm{N}_{2}$ flux, which will lead to stronger localization of carriers, and the uniformity of the quantum dots will reduce the defect density. The results demonstrate that lower $\mathrm{N}_{2}$ flow would improve the luminescence performance of InGaN QDs. The lifetime of samples S5 for fast decay is achieved as $450 \mathrm{ps}$ at $300 \mathrm{~K}$. The radiative lifetime is 910 ps on the assumption that the non-radiative recombination is fully suppressed at $10 \mathrm{~K}$, which is very close to the relatively good results of InGaN QDs or QW LEDs on c-plane substrates [12,22,28]. The results indicate that QDs can reduce the built-in electric field induced by polarizations and, thus, can result in shorter lifetime. Within the above analysis, it is concluded that the $\mathrm{N}_{2}$ flux should be minimized on the premise of N-rich condition for the formation of self-assembled InGaN QDs.

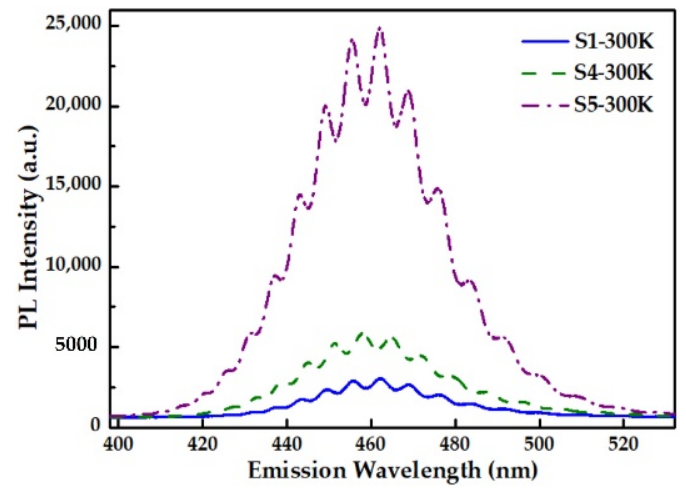

(a)

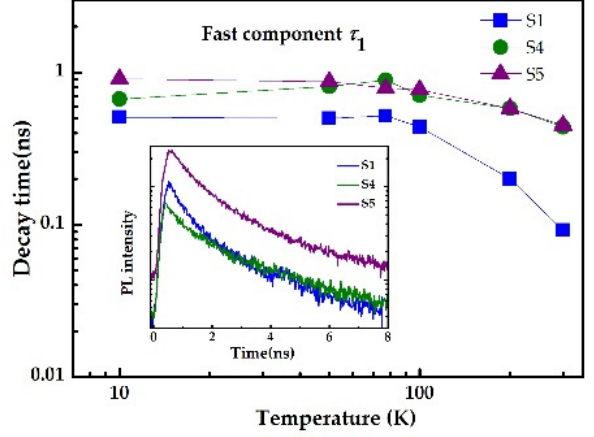

(b)

Figure 4. (a)The PL spectra of S1, S2 and S3 grown with varied $\mathrm{N}_{2}$ flow at room temperature; (b) the evolution of fast carrier lifetimes for sample S1, S4 and S5 with temperature. The inset displays PL decay curves at $10 \mathrm{~K}$ under excited power of $3 \mathrm{~mW}$.

For the self-assembled growth of InGaN QDs, growth temperature is undoubtedly another key parameter. High temperature can increase diffusion length of In and Ga ad-atom on the growth front and then promote QDs formation [29]. However, high temperature is not conducive to the incorporation of In and, hence, long-wavelength emission of InGaN material, because the evaporation of metal atoms would be enhanced significantly with increasing Tg. A group of samples S6, S7 and S8 have been grown at different $\mathrm{Tg}$, and the specific growth parameters have been listed in the Table 1 . The peak wavelengths of the three samples shown in Figure 5 are 462 nm, $523 \mathrm{~nm}$, and $581 \mathrm{~nm}$, respectively, and the insets are photographs of the resulting luminescence by S5 and S7. Apparently, the emission wavelengths of InGaN QDs have shown red shift with decreasing growth temperature. As previously mentioned, emission wavelength of InGaN QDs is very sensitive to the growth temperature. In addition, the truncated pyramidal-shaped feature of self-assembled QD structure with excellent independence is exhibited in blue InGaN QDs, which is better than longer-wavelength samples, according to 3D AFM images in Figure 2. It was confirmed that high temperature was beneficial to improving the micro-topography of InGaN QDs. In conclusion, Tg should be optimized to achieve long-wavelength emission without high evaporation rates, while ensuring sufficient diffusion length to improve crystal quality. 


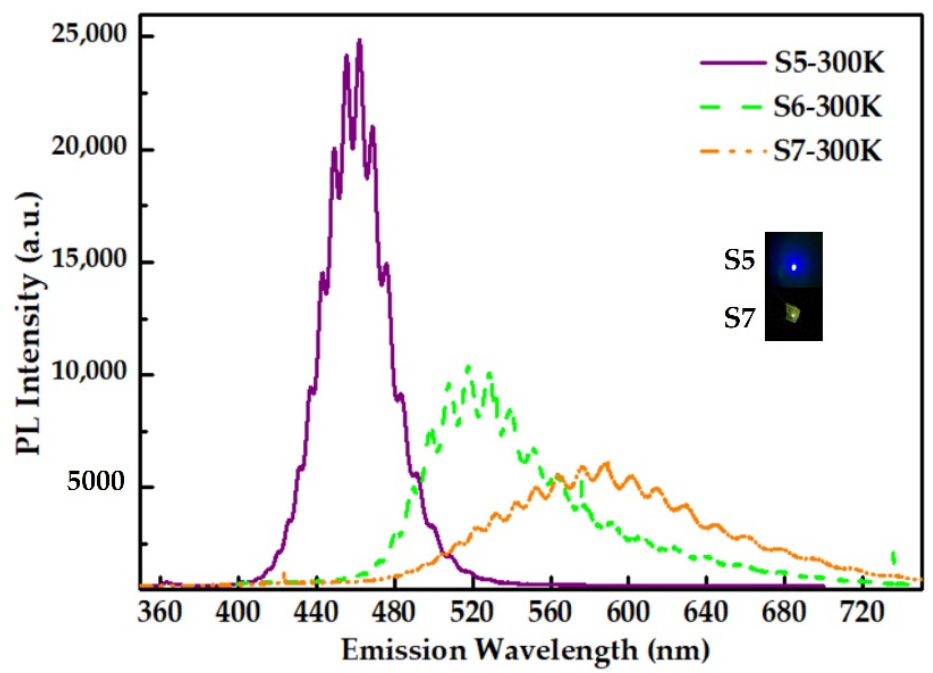

Figure 5. The PL spectra of S5, S6 and S7 grown with varied growth temperatures at room temperature and the insets are photographs of the resulting luminescence.

\subsection{Phase Diagram of InGaN QDs Grown by PA-MBE}

More InGaN QDs samples have been grown to probe into the growth conditions for the spontaneous formation of self-assembled InGaN QDs by SK mode. Based on above experiments and analysis results, we derived the phase diagram of InGaN QDs grown by PA-MBE, as illustrated in Figure 6, when the nitrogen flow rate and plasma power were fixed at $1.0 \mathrm{sccm}$ and $400 \mathrm{~W}$, respectively. In the first regime of phase diagram noted as Zone-I, low Tg and high total metal BEP led to high $\Delta \gamma$ and, thus, $\mathrm{E}_{3 \mathrm{D}}(\mathrm{h})>\mathrm{E}_{2 \mathrm{D}}(\mathrm{h})$, resulting in 2D surface growth of InGaN material. Furthermore, under high Ts and low metal BEP in Zone-III regime, it was difficult to from 3D islands because of high evaporation rates. Therefore, the growth window of QD islands was very narrow. More specifically, the metal $\mathrm{BEP}$ was less than $3 \times 10^{-8}$ torr, while growth temperature was lower than $630^{\circ} \mathrm{C}$.

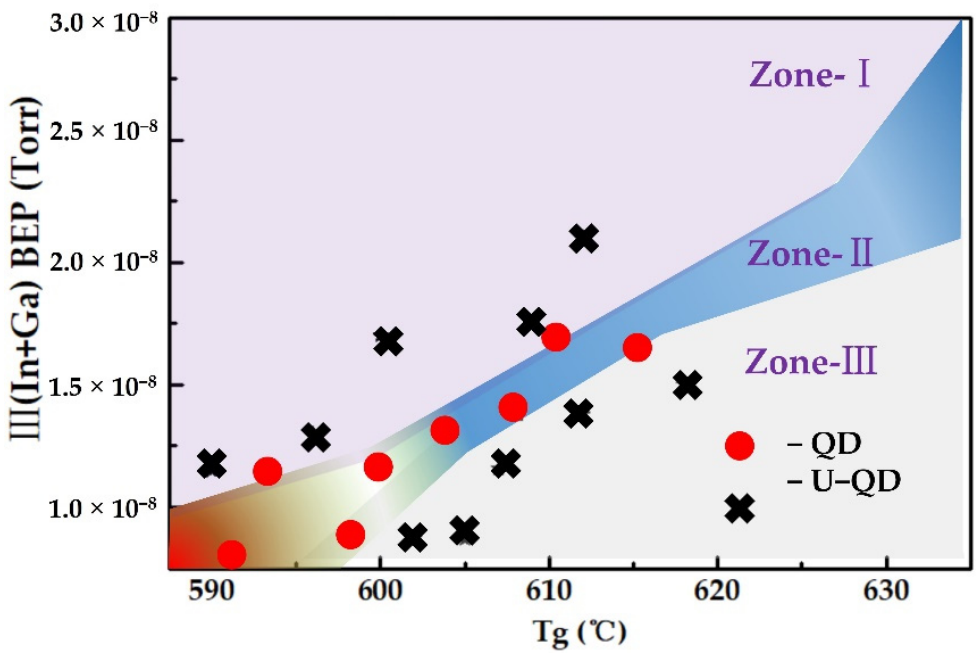

Figure 6. Phase diagram of InGaN quantum dot material growth.

\subsection{Nitrogen Modulation Epitaxy Method Based on SK Mode}

From the growth phase diagram of InGaN quantum dots grown by MBE, it can be seen that the window conditions for the growth of long-wavelength InGaN QDs were relatively narrow, so the growth method needed to be optimized. The nitrogen modulation epitaxy method is based on the SK mode, that is, the growth process is divided into two stages. The schematic diagram of the nitrogen modulation epitaxy method is shown in Figure 7a. In the first stage, it was ensured that a wetting layer was formed to realize 
the self-assembly growth of QDs under the condition of slight nitrogen enrichment, as shown in Figure 1. (1). This process also includes the RHEED diffraction pattern from the streaky- to spotty-like, that is, abrupt change from (2) to (3) in Figure 1. According to the monitoring of the samples' growth process, these stages usually lasted for about $4 \mathrm{~min}$. In the second stage, the nitrogen source is periodically turned on and off during the growth process, while the metal sources of In and Ga are always maintained. In the second stage, the nitrogen source must be periodically turned on and off. Turning on the nitrogen source ensured nitrogen-rich conditions for growing quantum dots in SK mode, while, when the nitrogen source was turned off, the reduction in the number of nitrogen atoms promoted the formation of 3D islands. At the same time, the metal atoms left on the growth surface had enough time to diffuse laterally, promoting the increase of In composition to red-shift the wavelength.

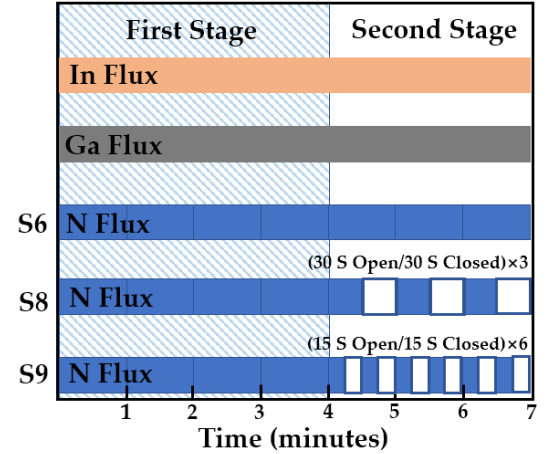

(a)

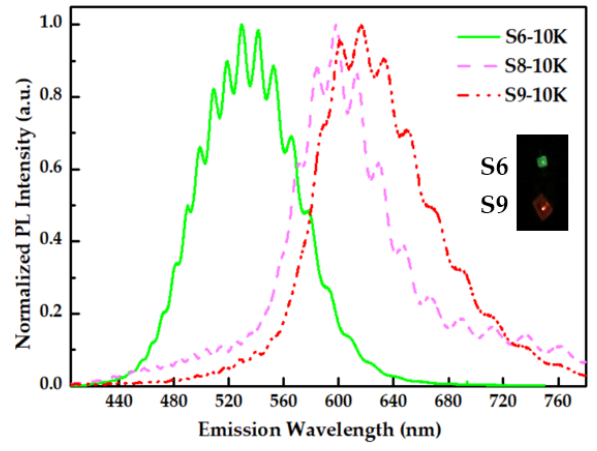

(b)

Figure 7. (a) Schematic diagram of nitrogen modulation epitaxy method; (b) The normalized PL emission spectrum of S6, S8, S9 at $10 \mathrm{~K}$ and the insets are photographs of the resulting luminescence.

The growth parameters of samples S6, S8 and S9 are listed in the Table 1 and the growth process is shown in Figure 7a; the three samples were kept the same in the first stage. The difference is that, in the second stage, the N source of sample S6 was always on, while the on and off times of sample $\mathrm{S} 8$ were $30 \mathrm{~s}$ for 3 cycles, respectively, while the on and off times of sample S9 were $15 \mathrm{~s}$ for 6 cycles. Figure 5 is the normalized PL emission spectrum of S6, S8, S9 at $10 \mathrm{~K}$, and the insets were photographs of the resulting luminescence by S6 and S9; in addition, the peak wavelengths were $523 \mathrm{~nm}, 598 \mathrm{~nm}$, and $622 \mathrm{~nm}$, respectively. The more cycles of the $\mathrm{N}$ source turned on and off, the better the wavelength redshift, so that the wavelength of InGaN QDs was shifted from green to red. Therefore, the nitrogen modulation epitaxy method was an effective solution for obtaining InGaN QDs emitting light in the red regions.

\subsection{Fabrication of InGaN QDs Green LED}

The schematic structure of InGaN QDs LED is shown in Figure 8a. A total of $450 \mathrm{~nm}$ Si-doped and $200 \mathrm{~nm} \mathrm{Mg-doped} \mathrm{GaN}$ were used as n-type and p-type contact layers, respectively. InGaN/GaN multilayers quantum dots (MQDs) structure with five pairs was employed as the active region of InGaN-based LED. GaN quantum barrier (QB) layers were grown at low temperature of $650{ }^{\circ} \mathrm{C}$ to protect InGaN QDs. A $22 \mathrm{~nm}$-thick AlGaN layer was applied as electron blocking layer (EBL) to reduce electron leakage. Figure $8 \mathrm{~b}$ shows the cross-sectional TEM image of InGaN/GaN MQDs structure in QDs LED. We could clearly observe that distinct InGaN QDs were scattered in GaN barrier layers. The height of the QD marked in red circle was about $2.6 \mathrm{~nm}$. In addition, the thickness of the GaN QB layer was about $19.4 \mathrm{~nm}$, and the InGaN/GaN interface was very abrupt. Afterwards, an InGaN-based LED was fabricated by using conventional LED process. However, it should be noted that current spreading layer was not introduced in device fabrication. Finally, the epilayers were divided into devices with $250 \mu \mathrm{m} \times 250 \mu \mathrm{m}$. 


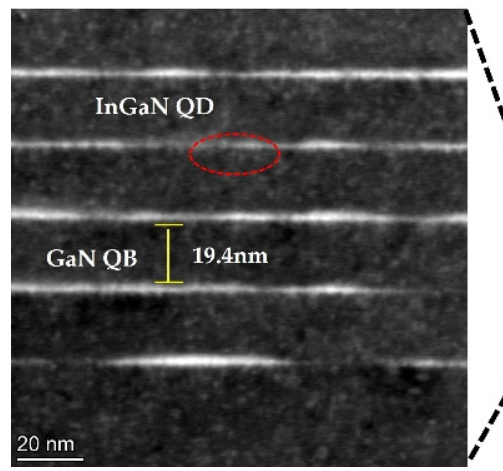

(b)

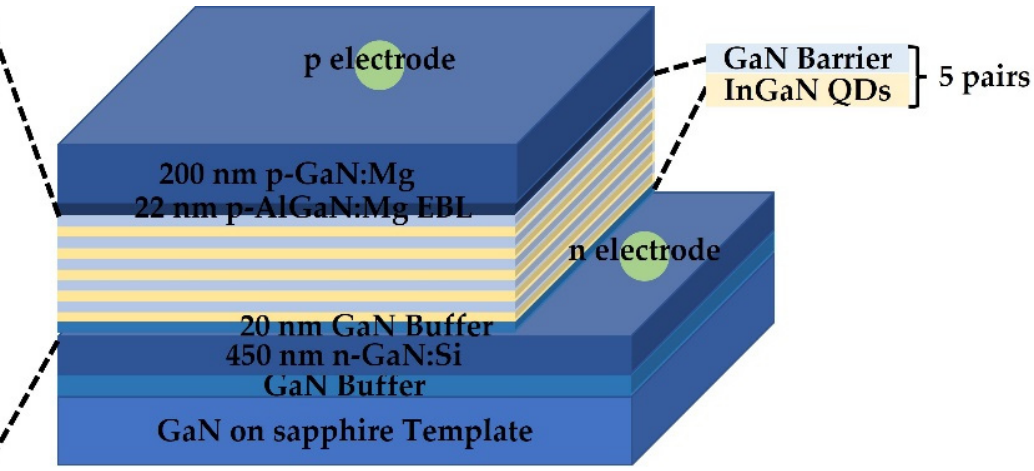

(a)

Figure 8. (a) Schematic diagram of the QD LED structure and (b) Cross-sectional TEM image of InGaN/GaN MQDs region in LED.

Figure 9a,b show the variations of forward voltage, light output power (LOP) and external quantum efficiency (EQE), respectively, with injection current from $5 \mathrm{~mA}$ to $100 \mathrm{~mA}$, i.e., corresponding current density from $8 \mathrm{~A} / \mathrm{cm}^{2}$ to $160 \mathrm{~A} / \mathrm{cm}^{2}$. The LOP increased firstly and then tended to be stabilized gradually with the increase of injection current. The QD LED presented a significant efficiency droop; the efficiency of QD LED was as low as $0.14 \%$ at injection current of $5 \mathrm{~mA}$. The efficiency droop at high current owed to the large non-radiative recombination rate. We inferred that poor performance may be attributed to following possible reasons. One is that thick GaN QB layers grown at low temperature produced high-density defects, which resulted in nonradiative recombination, as well as carrier delocalization [30,31]. The other is that severe current crowding (CC) occurring in $\mathrm{p}-\mathrm{GaN}$ without current spreading layer enhanced Auger recombination and electron leakage [32], which are underling mechanisms of the efficiency recombination in lateral GaN-based LEDs [30,33]. Furthermore, because QDs have lower electron capture cross section that QWs, the low capture probability would reduce the efficiency of LED. In addition, electroluminescence (EL) measurements under various injection current at room temperature were performed on the LED. As depicted in Figure 9c,d, the peak wavelength located at $505 \mathrm{~nm}$ with a shift of less than $1.0 \mathrm{~nm}$ as the injection current was gradually increased from $5 \mathrm{~mA}$ to $100 \mathrm{~mA}$. It can be compared with green semipolar InGaN QW LEDs [34]. The results showed excellent wavelength stability of LED, which was mainly attributed to the fully screened polarization-related electric field and, hence, to suppressed QCSE in InGaN QDs $[12,35]$. The epitaxial structure and fabrication process would be further optimized in our future work to overcome efficiency droop and to improve the performance of InGaN-based green LEDs.
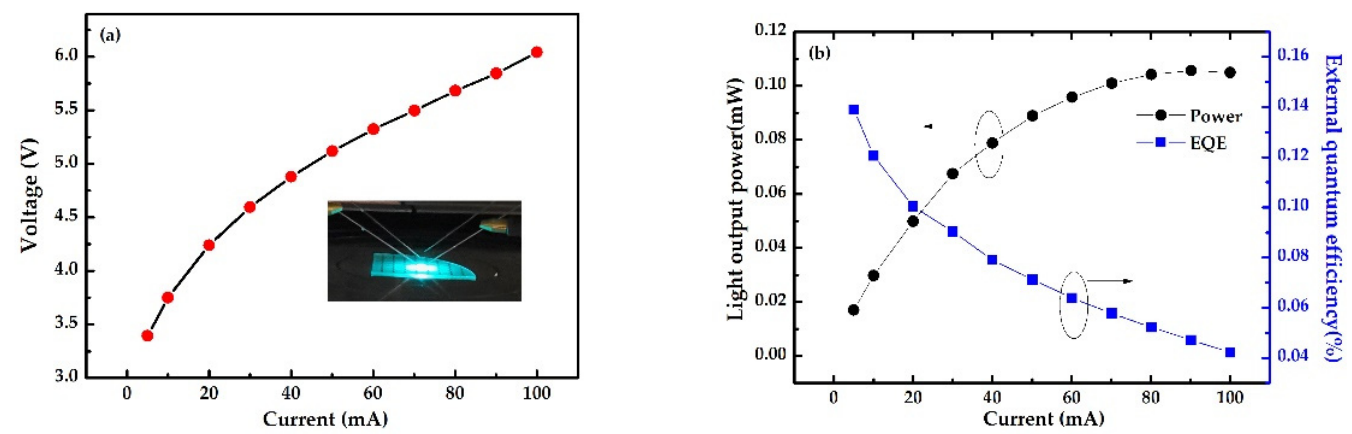

Figure 9. Cont. 

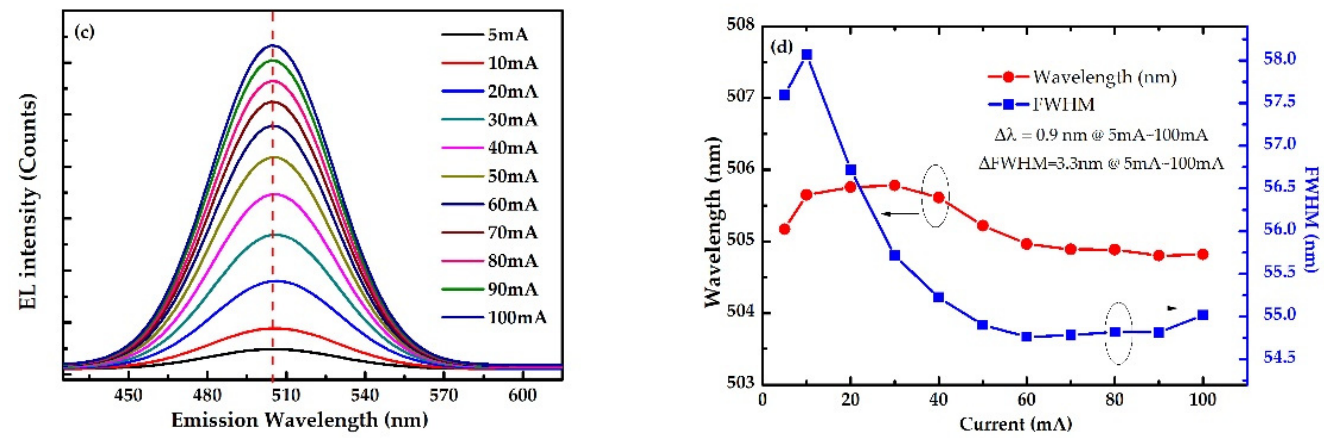

Figure 9. (a) Forward voltage versus injection current; (b) LOP and EQE versus injection current; (c) EL spectra of QD LED under injection current from $5 \mathrm{~mA}$ to $100 \mathrm{~mA}$; (d) the variation of EL peak wavelength and FWHM with injection current.

\section{Conclusions}

In summary, we explored S-K growth of self-assembled InGaN QDs on GaN-onsapphire templates using PA-MBE by regulating $\mathrm{In} / \mathrm{Ga}$ flux ratio, $\mathrm{N}_{2}$ flow and growth temperature. The effects of growth parameters on the spontaneous formation of InGaN QDs were investigated by AFM and PL measurements; consequently, the phase diagram of MBE-grown InGaN QDs was described. Small $\mathrm{N}_{2}$ flow is instrumental in improving morphology and luminescent properties of InGaN QDs, whereas low growth temperature and In/Ga flux ratio are applicable for long-wavelength emission. As a result, InGaN QDs with light emission from $460 \mathrm{~nm}$ to $622 \mathrm{~nm}$ were achieved by tuning growth conditions and with nitrogen modulation epitaxy method. In addition, a $505 \mathrm{~nm}$ green LED based on InGaN/GaN MQDs was prepared. The LED had a low EQE of less than $0.14 \%$ and showed a strong efficiency droop with increasing injection current. However, the EL emission wavelength exhibited a minuscule shift of less than $1 \mathrm{~nm}$ as injection current was increased from $5 \mathrm{~mA}$ to $100 \mathrm{~mA}$, which indicated that the built-in filed was screened well in InGaN QDs.

Author Contributions: Conceptualization and methodology, X.Z. and W.Y.; formal analysis and visualization, Z.X., H.Q., Y.S., S.K., Y.M., S.I. and Y.N.; investigation, Y.G.; writing original draft preparation, X.Z.; writing review and editing, W.Y.; supervision, A.T.; project administration, Y.C.; funding acquisition, S.L. and L.B. All authors have read and agreed to the published version of the manuscript.

Funding: This research was funded by the National Natural Science Foundation of China (Grant Nos. 61804163, 61875224, and 61827823), the Key R\&D Program of Jiangsu Province (Grant No. BE2021008-4), Key Research Program of Frontier Sciences, CAS (No. ZDBS-LY-JSC034). This research was also funder by Project in Science and Technique Plans of Huzhou (No. 2021GZ04), SINANO (No. Y8AAQ21001) and Vacuum Interconnected Nanotech Workstation (Nano-X, No. B2006).

Institutional Review Board Statement: Not applicable.

Informed Consent Statement: Not applicable.

Data Availability Statement: The data presented in this study are available within the manuscript.

Acknowledgments: The authors are thankful for the technical support from the Platform Characterization \& Test and the Nanofabrication Facility of Suzhou Institute of Nano-Tech and Nano-Bionics (SINANO), Chinese Academy of Sciences (CAS).

Conflicts of Interest: The authors declare no conflict of interest.

\section{References}

1. Liu, Z.; Lin, C.H.; Hyun, B.R.; Sher, C.W.; Lv, Z.; Luo, B.; Jiang, F.; Wu, T.; Ho, C.-H.; Kuo, H.-C.; et al. Micro Light Emitting Diodes with Quantum Dots in Display Technology. Light Sci. Appl. 2020, 9, 1-23. [CrossRef] [PubMed]

2. Wierer Jr, J.J.; Tansu, N. III-Nitride Micro-Leds for Efficient Emissive Displays. Laser Photonics Rev. 2019, 13, 1900141. [CrossRef] 
3. Wang, L.; Wang, L.; Yu, J.; Hao, Z.; Luo, Y.; Sun, C.; Han, Y.J.; Xiong, B.; Wang, J.; Li, H.T. Abnormal Stranski-Krastanov Mode Growth of Green InGaN Quantum Dots: Morphology, Optical Properties, and Applications in Light Emitting Devices. ACS Appl. Mater. Interfaces 2019, 11, 1228-1238. [CrossRef] [PubMed]

4. Frost, T.; Su, G.L.; Hazari, A.; Dallesasse, J.M.; Bhattacharya, P. Red and near-Infrared III-Nitride Quantum Dot Lasers. IEEE J. Sel. Top. Quantum Electron. 2017, 23, 1-9. [CrossRef]

5. Bulashevich, K.A.; Karpov, S.Y. Impact of Surface Recombination on Efficiency of III-Nitride Light Emitting Diodes. Phys. Status Solidi-Rapid Res. Lett. 2016, 10, 480-484. [CrossRef]

6. Li, P.P.; Zhao, Y.B.; Li, H.J.; Che, J.M.; Zhang, Z.H.; Li, Z.C.; Zhang, Y.Y.; Wang, L.C.; Liang, M.; Yi, X.Y.; et al. Very High External Quantum Efficiency and Wall-Plug Efficiency 527nm InGaN Green Leds by MOCVD. Opt. Express 2018, 26, 33108-33115. [CrossRef]

7. Jiang, X.; Zheng, C.; Mo, C.; Wang, X.; Zhang, J.; Quan, Z.; Liu, J.L.; Jiang, F.Y. Study on the Performance of InGaN-Based Green Led by Designing Different Preparing Layers. Opt. Mater. 2019, 89, 505-511. [CrossRef]

8. Lv, Q.; Liu, J.; Mo, C.; Zhang, J.; Wu, X.; Wu, Q.; Jiang, F. Realization of Highly Efficient InGaN Green Leds with Sandwich-Like Multiple Quantum Well Structure: Role of Enhanced Interwell Carrier Transport. ACS Photonics 2019, 6, 130-138. [CrossRef]

9. Royo, P.; Stanley, R.P.; Ilegems, M.; Streubel, K.; Gulden, K.H. Experimental Determination of the Internal Quantum Efficiency of AlGaInP Microcavity Light Emitting Diodes. J. Appl. Phys. 2002, 91, 2563-2568. [CrossRef]

10. Boroditsky, M.; Gontijo, I.; Jackson, M.; Vrijen, R.; Yablonovitch, E.; Krauss, T.; Cheng, C.C.; Scherer, A.; Bhat, R.; Krames, M. Surface Recombination Measurements on III-V Candidate Materials for Nanostructure Light-Emitting Diodes. J. Appl. Phys. 2000, 87, 3497-3504. [CrossRef]

11. Wang, Y.; Duan, B.; Deng, G.; Yu, Y.; Niu, Y.; Yu, J.; Ma, H.; Shi, Z.; Zhang, B.; Zhang, Y. The Study of Properties of Blue-Green InGaN/GaN Multiple Quantum Wells Grown at Different Pressures. Superlattices Microstruct. 2021, 153, 106863. [CrossRef]

12. Samuel, B.; Cooper, D.; Rochat, N.; Mavel, A.; Barbier, F.; Dussaigne, A. Origins of Nanoscale Emission Inhomogeneities of High Content Red Emitting InGaN/InGaN Quantum Wells. J. Appl. Phys. 2021, 129, 173105. [CrossRef]

13. Srivastava, S.K.; Pramanik, M.; Palit, D.; Mathur, B.K.; Kar, A.K.; Ray, B.S.; Haeuseler, H.; Cordes, W. Electrical, Optical, and Scanning Tunneling Microscopic Studies on Layer Type CdIn $\mathrm{S}_{4-\mathrm{x}} \mathrm{Se}_{\mathrm{x}}(1.75 \leq \mathrm{x} \leq 2.75)$. Chem. Mater. 2001, 13, $4342-4347$. [CrossRef]

14. Zhao, C.; Tang, C.W.; Lai, B.; Cheng, G.; Wang, J.; Lau, K.M. Low-Efficiency-Droop InGaN Quantum Dot Light Emitting Diodes Operating in the "Green Gap". Photonics Res. 2020, 8, 750-754. [CrossRef]

15. Schulz, S.; O'Reilly, E.P. Theory of Reduced Built-in Polarization Field in Nitride-Based Quantum Dots. Phys. Rev. B 2010, 82, 033411. [CrossRef]

16. Wu, Y.-R.; Lin, Y.-Y.; Huang, H.-H.; Singh, J. Electronic and Optical Properties of Ingan Quantum Dot Based Light Emitters for Solid State Lighting. J. Appl. Phys. 2009, 105, 013117. [CrossRef]

17. Wang, L.; Yang, D.; Hao, Z.B.; Luo, Y. Metal-Organic-Vapor Phase Epitaxy of InGaN Quantum Dots and Their Applications in Light-Emitting Diodes. Chin. Phys. B 2015, 24, 067303. [CrossRef]

18. Banerjee, A.; Frost, T.; Jahangir, S.; Stark, E.; Bhattacharya, P. InGaN/GaN Self-Organized Quantum Dot Lasers Grown by Molecular Beam Epitaxy. J. Cryst. Growth 2013, 378, 566-570. [CrossRef]

19. Lv, W.; Wang, L.; Wang, J.; Xing, Y.; Zheng, J.; Yang, D.; Hao, Z.; Luo, Y. Green and Red Light-Emitting Diodes Based on Multilayer InGaN/GaN n Dots Grown by Growth Interruption Method. Jpn. J. Appl. Phys. 2013, 52, 08JG13. [CrossRef]

20. Wei, X.; Al Muyeed, S.A.; Xue, H.; Palmese, E.; Song, R.; Tansu, N.; Wierer, J.J. Near-Infrared Electroluminescence of Algan Capped InGaN Quantum Dots Formed by Controlled Growth on Photoelectrochemical Etched Quantum Dot Templates. Photonics Res. 2022, 10, 33-40. [CrossRef]

21. Iida, D.; Ohkawa, K. Recent Progress in Red Light-Emitting Diodes by III-Nitride Materials. Semicond. Sci. Technol. 2022, 37, 013001. [CrossRef]

22. Cho, I.W.; Lee, B.; Ryu, M.Y.; Lee, K.; Kim, J.S. Luminescence Properties of InGaN/GaN Light-Emitting Diodes with Violet, Blue, and Green Emission. J. Korean Phys. Soc. 2021, 78, 275-279. [CrossRef]

23. Dussaigne, A.; Barbier, F.; Damilano, B.; Chenot, S.; Grenier, A.; Papon, A.M.; Samuel, B.; Ben Bakir, B.; Vaufrey, D.; Pillet, J.C.; et al. Full InGaN Red Light Emitting Diodes. J. Appl. Phys. 2020, 128, 135704. [CrossRef]

24. Mariette, H. Formation of Self-Assembled Quantum Dots Induced by the Stranski-Krastanow Transition: A Comparison of Various Semiconductor Systems. Comptes Rendus Phys. 2005, 6, 23-32. [CrossRef]

25. Hums, C.; Finger, T.; Hempel, T.; Christen, J.; Dadgar, A.; Hoffmann, A.; Krost, A. Fabry-Perot Effects in InGaN/GaN Heterostructures on Si-Substrate. J. Appl. Phys. 2007, 101, 033113. [CrossRef]

26. Kumagai, Y.; Kikuchi, J.; Matsuo, Y.; Kangawa, Y.; Tanaka, K.; Koukitu, A. Thermodynamic Analysis of Inn and In $\mathrm{In}_{1-\mathrm{X}} \mathrm{N}$ Movpe Using Various Nitrogen Sources. J. Cryst. Growth 2004, 272, 341-347. [CrossRef]

27. Liu, L.; Wang, L.; Liu, N.; Yang, W.; Li, D.; Chen, W.; Feng, Z.C.; Lee, Y.C.; Ferguson, I.; Hu, X. D Investigation of the Light Emission Properties and Carrier Dynamics in Dual-Wavelength InGaN/GaN Multiple-Quantum Well Light Emitting Diodes. J. Appl. Phys. 2012, 112, 083101. [CrossRef]

28. Zhao, C.; Tang, C.W.; Cheng, G.; Wang, J.; Lau, K.M. InGaN Quantum Dots with Short Exciton Lifetimes Grown on Polar C-Plane by Metal-Organic Chemical Vapor Deposition. Mater. Res. Express 2020, 7, 115903. [CrossRef] 
29. Damilano, B.; Demolon, P.; Brault, J.; Huault, T.; Natali, F.; Massies, J. Blue-Green and White Color Tuning of Monolithic Light Emitting Diodes. J. Appl. Phys. 2010, 108, 073115. [CrossRef]

30. Cho, J.; Schubert, E.F.; Kim, J.K. Efficiency Droop in Light-Emitting Diodes: Challenges and Countermeasures. Laser Photonics Rev. 2013, 7, 408-421. [CrossRef]

31. Hader, J.; Moloney, J.V.; Koch, S.W. Temperature-Dependence of the Internal Efficiency Droop in GaN-Based Diodes. Appl. Phys. Lett. 2011, 99, 181127. [CrossRef]

32. Malyutenko, V.K.; Bolgov, S.S.; Podoltsev, A. Current Crowding Effect on the Ideality Factor and Efficiency Droop in Blue Lateral InGaN/GaN Light Emitting Diodes. Appl. Phys. Lett. 2010, 97, 251110. [CrossRef]

33. Verzellesi, G.; Saguatti, D.; Meneghini, M.; Bertazzi, F.; Goano, M.; Meneghesso, G.; Zanoni, E. Efficiency Droop in InGaN/GaN Blue Light-Emitting Diodes: Physical Mechanisms and Remedies. J. Appl. Phys. 2013, 114, 071101. [CrossRef]

34. Zhao, Y.; Oh, S.H.; Wu, F.; Kawaguchi, Y.; Tanaka, S.; Fujito, K.; Speck, J.S.; Denbaars, S.; Nakamura, S. Green Semipolar (2021) InGaN Light-Emitting Diodes with Small Wavelength Shift and Narrow Spectral Linewidth. Appl. Phys. Express 2013, 6, 062102. [CrossRef]

35. Lv, W.; Wang, L.; Wang, L.; Xing, Y.; Yang, D.; Hao, Z.; Luo, Y. InGaN Quantum Dot Green Light-Emitting Diodes with Negligible Blue Shift of Electroluminescence Peak Wavelength. Appl. Phys. Express 2014, 7, 025203. [CrossRef] 